Parcours de recherche en SIC: littérature scientifique, méthodes et terrain

\title{
Imaginaire social du Vietnam contemporain à la lumière des Sciences de l'Information et de la Communication
}

The Social Imaginary of Contemporary Vietnam in Light of the Information and Communication Sciences

\section{Anh-Ngoc Hoang}

\section{CpenEdition}

Journals

Édition électronique

URL : http://journals.openedition.org/edc/894

DOI : $10.4000 /$ edc. 894

ISSN : 2101-0366

\section{Éditeur}

Université Lille-3

\section{Édition imprimée}

Date de publication : 1 avril 2009

Pagination : 81-96

ISBN : 978-2-917562-01-7

ISSN : 1270-6841

\section{Référence électronique}

Anh-Ngoc Hoang, «Imaginaire social du Vietnam contemporain à la lumière des Sciences de I'Information et de la Communication ». Études de communication [En ligne], 32 | 2009, mis en ligne le 01 avril 2011, consulté le 05 mai 2019. URL : http://journals.openedition.org/edc/894 ; DOI : 10.4000/ edc. 894

Ce document a été généré automatiquement le 5 mai 2019.

(c) Tous droits réservés 


\title{
Imaginaire social du Vietnam contemporain à la lumière des Sciences de l'Information et de la Communication
}

\author{
The Social Imaginary of Contemporary Vietnam in Light of the Information and \\ Communication Sciences
}

Anh-Ngoc Hoang

Cette communication provient d'un travail de thèse en cours ${ }^{1}$. Loin de prétendre par conséquent à un quelconque résultat définitif, je me contente de livrer certaines réflexions de jeune chercheur au milieu de son parcours de recherche en SIC, où s'entrecroisent intuitions et hypothèses, enthousiasme et désorientation, acquis et défis. $\mathrm{Au}$ cœur de cette étape de construction de mon objet de recherche, se posent de façon aiguë les questionnements d'ordre épistémologique : en quoi l'approche méthodologique info-communicationnelle permettrait-elle d'apporter un éclairage neuf sur une vieille question de l'identité culturelle vietnamienne sous l'angle d'un imaginaire social? Alors, pour moi, questionner la reconfiguration identitaire du Vietnam contemporain ne va pas in fine sans questionner l'identité des SIC en termes de posture épistémologique. Mon article comporte deux grandes parties. D'abord, je présenterai les grandes lignes de mon projet de thèse : sa problématique et ses hypothèses de recherche. Ensuite, j'essaierai d'expliciter la construction de cette problématique de recherche d'un point de vue méthodologique en SIC, les raisons de mes choix méthodologiques et les types de connaissances auxquels ils permettront l'accès. 


\section{Imaginaire social du Vietnam contemporain : une cyber-vietnamité}

2 Ma recherche part d'une hypothèse centrale selon laquelle l'imagination collective en tant que pratique sociale permet aujourd'hui une reconstruction ou une reconfiguration identitaire du Vietnam contemporain dans un monde caractérisé par le développement exponentiel de technologies d'information et de communication (TIC) et les flux croissants de personnes favorisés par la globalisation. Ainsi la construction d'un imaginaire social du Vietnam est à l'œuvre aujourd'hui, effectuée dans les liens entre les Vietnamiens nationaux et la diaspora, et par le biais des TIC. J'appelle cet imaginaire social la cyber-vietnammité, c'est-à-dire "un ensemble de discours symboliques» (Ricœur, 1991, p. 474), construit par les Vietnamiens eux-mêmes (ceux du Vietnam et ceux de la diaspora) pour se donner une image de soi, se représenter en tant que groupe social, par le biais des TIC. La question de recherche principale pourrait donc s'énoncer en ces termes: "Comment les Vietnamiens nationaux et les Vietnamiens diasporiques construisent, à travers des liens établis entre eux, et par le biais des TIC, une cybervietnamité?». Afin d'appréhender le processus de construction de cette cybervietnamité, j'avance les quatre hypothèses suivantes :

- les TIC rendent possibles et multiplient les liens actuels entre le Vietnam et la diaspora dispersée dans plusieurs lieux géographiques et construisent ainsi un transnationalisme vietnamien contemporain ;

- la construction de ces cyber-liens transnationaux s'effectue grâce à une pratique imaginative sociale déployée dans les TIC, notamment les web, forums de discussion, weblogs ;

- cette imagination sociale à l'œuvre dans les cyber-liens transnationaux entre les Vietnamiens nationaux et la diaspora produit un ensemble de discours symboliques ou un imaginaire social à double dimension: intégrative, identitaire d'une part et subversive, inventive d'autre part ;

- prenant forme dans des flux transnationaux contextualisés, cet imaginaire social reste discipliné par des logiques de pouvoir, soumis aux structures socio-politico-économiques nationales et globales, et déterminé par différents dispositifs mis en place.

\section{Construction du dispositif méthodologique}

3 Ayant adopté une posture épistémologique, je me suis appuyé sur un acquis, largement partagé par la communauté scientifique, quant à la spécificité des sciences de l'information et de la communication en tant que discipline: ces sciences prennent en compte, d'une manière particulière, l'articulation de trois dimensions de nature différente d'un objet de la réalité, à savoir celle du sens, celle du social et celle de la technique, de manière à en faire une cohérence logique et théorique (Ollivier, 2007, p. 169). Ces dimensions font partie intégrante des objets de recherche scientifiques construits en SIC qui sont fondamentalement composites et hétérogènes. Il en est ainsi pour les phénomènes culturels et identitaires vietnamiens auxquels s'intéresse ma recherche. En effet, interroger la construction de l'imaginaire social du Vietnam contemporain suppose de poser les questions des acteurs, des dispositifs et du sens. 


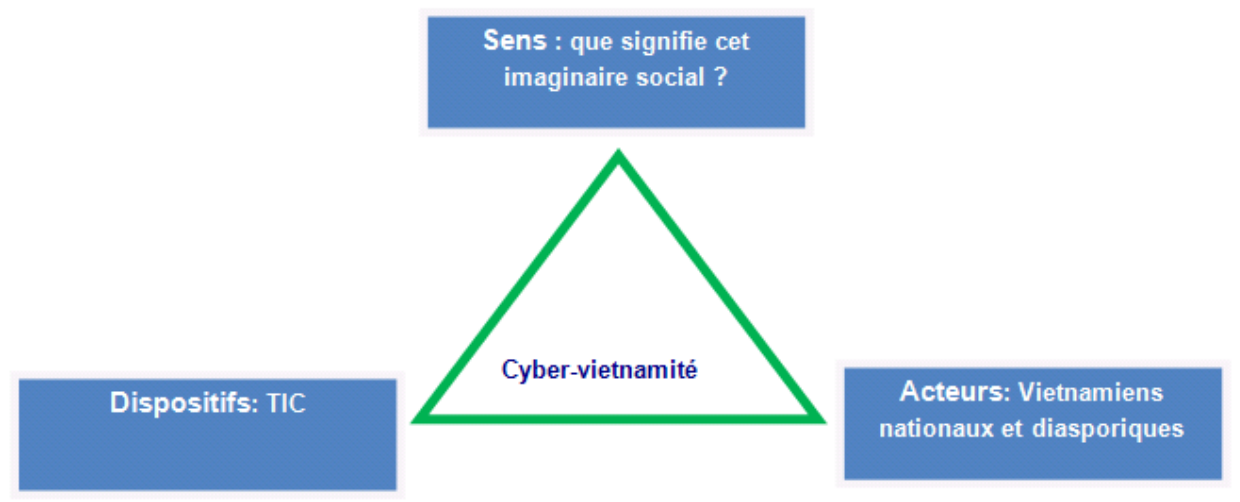

Une triple approche du processus de construction de la cyber-vietnamité

4 En d'autres termes, ma problématique de recherche s'articulera autour des approches suivantes: une approche actancielle (des Vietnamiens nationaux et diasporiques), une approche logistique (technologies de communication comme dispositifs de construction) et une approche herméneutique (quels sens donnés à cet imaginaire social?). La prise en compte de cette triple dimension des phénomènes imaginaires servira de fil conducteur qui caractérisera mes choix méthodologiques. Dans la partie qui suit, j'argumenterai comment ces choix méthodologiques permettraient d'avoir accès à un certain type de regard sur une réalité examinée, donc à un certain type de savoir scientifique disciplinairement construit. Cette réflexion méthodologique est nourrie des exemples de mon corpus. Pour la clarté du propos, j'ai choisi de présenter séparément chacune de trois dimensions, à savoir acteurs, dispositifs et sens, de façon à mettre en avant son caractère structurant et à souligner aussi une vision globale de ces trois dimensions par rapport à la question de la construction méthodologique.

\section{Question des acteurs}

\section{Nécessité d'une étude historique et terminologique}

En s'intéressant aux liens établis entre les Vietnamiens nationaux et les Vietnamiens diasporiques, mon projet prend en considération le rôle que jouent ces différents acteurs dans le cadre de leurs échanges via les TIC. Cette question des acteurs pose d'emblée une problématique sur la pertinence des termes désignant diverses populations vietnamiennes résidant à l'étranger. C'est pourquoi, j'ai choisi de consacrer une partie de l'analyse du corpus à une étude historique et terminologique de la communauté vietnamienne expatriée. Ce regard historique, documenté sur la base des travaux sociologiques $^{2}$, anthropologiques ${ }^{3}$ et historiques ${ }^{4}$ cherche à situer les communautés vietnamiennes expatriées contemporaines dans une histoire plus longue du Vietnam. Cette lecture dans l'épaisseur historique éviterait le risque d' « actualisme » que court une thèse en SIC comme la mienne qui prend pour objet un phénomène assez récent qu'est la migration en masse des Vietnamiens après l'événement de 1975. Ce cadrage historique favorise également une double lecture des phénomènes de circulation des Vietnamiens à l'ère de la globalisation : continuité et ruptures. Sur le plan de continuité, on verra que le mouvement migratoire des Vietnamiens à la fin du $\mathrm{XX}^{\mathrm{e}}$ siècle s'inscrit dans une longue histoire de déplacements de ce peuple à travers des vicissitudes historiques ${ }^{5}$. Quant aux 
ruptures, elles sont dues aux caractéristiques propres à cette migration massive des Vietnamiens contemporains qui ont formé des «Viet kieu» ou une "diaspora vietnamienne ». Par ailleurs, un examen détaillé de la constitution de la diaspora vietnamienne contemporaine nous donnera aussi une connaissance factuelle et chiffrée de cette réalité. La question de terminologie quant à la façon de désigner cette population vietnamienne spécifique se pose aussi de façon complexe. Est de taille l'enjeu d'un terme « approprié » qui puisse traduire " fidèlement » une réalité hétérogène. J'en donnerai les cinq expressions les plus courantes en essayant d'expliquer ce que l'on entend par chacune d'elles et j'indiquerai par la suite mon choix terminologique. D'abord, l'expression "Viet kieu» est un mot courant en sino-vietnamien, par lequel les Vietnamiens vivant dans leur pays désignent leurs compatriotes résidant à l'étranger. «Viet kieu » est modelé sur une expression chinoise. En effet, « kieu » tire son origine du terme chinois "qiao" (second tone) qui signifie "vivre à l'étranger» ou "personne vivant à l'étranger ». "Viet kieu » est donc semblable au terme chinois « huaqiao » qui se réfère aux Chinois vivant à l'étranger ${ }^{6}$. Mais, selon certains linguistes vietnamiens, «le caractère « kieu » représente un séjourneur, un visiteur temporaire, et dans le contexte vietnamien, quelqu'un qui dépend de l'œuvre de bienfaisance d'un autre pays $»^{7}$. Cependant, l'expression « Viet kieu » peut avoir aussi une connotation négative à la fois auprès des Vietnamiens nationaux, des autochtones et des Vietnamiens diasporiques euxmêmes. En effet, pour les Vietnamiens nationaux, l'expression «Viet kieu " véhicule certains stéréotypes qui caractérisent "Viet kieu» comme tapageur, arrogants, dépensiers et bourgois gentilhomme. Les Américains peuvent considérer les Viet kieu comme des Américains d'origine vietnamienne (Vietnamese-Americans) «simplistement anti-communistes et matérialistes $»^{8}$. En diaspora, notamment dans l'univers intellectuel et universitaire, "Viet kieu» dégageant un "goût déplaisant ", la tendance semble substituer à «Viet kieu » d'autres termes comme « Kieu bao » ou « Đồng bào hải ngoại » (« compatriotes d'outre-mer», " compatriotes expatriés», " compatriotes vivant à l'étranger »). L'expression « Viet kieu » est entrée aussi, pour un temps, dans des discours officiels de la propagande du gouvernement vietnamien. Pendant la guerre, il y avait "Association de Viet kieu patriotes $»^{10}$ en France, au Canada, aux Etats-Unis, etc. qui rassemblaient des intellectuels vietnamiens de gauche vivant à l'étranger. Les deux expressions «Vietnamiens d'outre-mer» (Overseas Vietnamese) et «Vietnamiens résidant à l'étranger » ne traduisent qu'une dimension de l'espace physique. Il est intéressant de noter que les versions en anglais et en français de derniers textes gouvernementaux adoptent ces expressions : en anglais, la Résolution No 36, datée de 2004 utilise les termes "Overseas Vietnamese " ${ }^{11}$; la Décision No 135, datée de 2007, "Vietnamese residing overseas $»^{12}$. En français, ces deux textes adoptent l'expression "Vietnamiens résidant à l'étranger $»^{13}$. Le choix de ces expressions assez neutres (seul l'élément de la dispersion dans l'espace physique est pris en compte) traduit certes une volonté du gouvernement vietnamien de ne pas évoquer toute connotation très chargée historiquement et politiquement qui pourrait exister dans d'autres termes. L'expression «Vietnamiens expatriés » (Vietnamese expatriates) souligne seulement la dispersion, dans un sens unique, de la population vietnamienne. Notons que ces deux expressions, à savoir "Overseas Vietnameses » et « Vietnamese expatriates » sont souvent utilisées indifféremment pour traduire l'expression vietnamienne « Cộng đồng người Việt hải ngoại » en usage courant dans le parler des communautés vietnamiennes diasporiques anglophones. Quant à l'expression "Réfugiés vietnamiens》, ou "Réfugiés vietnamiens du régime communiste", le terme "n'évoque pas seulement, pour la plupart des Vietnamiens vivant à 
l'étranger, la condition de l'exil, mais connote aussi le rêve d'un pays imaginaire, une sorte de paradis perdu, et une volonté de restauration $»^{14}$. Selon L.-J. Dorais, seule une minorité de Viêt Kiêu de première génération, ayant quitté le Vietnam à l'âge adulte, souvent anciens militaires ou cadres politiques de la République du Vietnam et engagés dans des mouvements politiques anti-communistes se définit encore comme réfugiée, plus de 30 ans après les événements de 1975. En ce qui concerne la dernière expression, «diaspora vietnamienne ", elle provoque beaucoup de débat, car le terme "diaspora » lui-même reste très discuté en sciences sociales depuis plus d'une dizaine d'années ${ }^{15}$. L'usage initial de l'expression "diaspora vietnamienne » a été peut-être effectué par des Vietnamiens catholiques d'outre-mer ${ }^{16}$. Un récent programme de recherche de trois ans, mené par le William Joiner Center qui relève de Massachusetts University (États-Unis) a été intitulé " (Re)Constructing Identity and Place in the Vietnamese Diaspora ». En 2003, le moteur de recherche de Yahoo a affiché 900 résultats contenant le terme "Vietnamese Diaspora ${ }^{17}$. Pour l'anthropologue L.-J. Dorais, spécialiste de la communauté des Vietnamiens au Canada, si l'on partage les critères de définition de «diaspora » proposés par Khachig Tölölyan ${ }^{18}$, les Viet kieu canadiens «ne constituent pas de diaspora à proprement parler, mais leur histoire possède une dimension diasporique certaine» (Dorais, 2001, p. 23). Nguyen Ba Tung, chercheur aux Etats-Unis, s'appuyant sur une conception de « diaspora » proposée par F. Riggs, souligne ses deux caractères « informel » et « actif ». Il soutient qu'environ deux millions de Vietnamiens d'outre-mer dispersés aujourd'hui dans le monde constituent une véritable "diaspora vietnamienne » et qu'elle se distingue d'autres formes de communautés transnationales (travailleurs invités, étudiants à l'étranger, diplomates...). Il convient donc de signaler toute la difficulté que suscite le souhait de couvrir avec une seule notion une pluralité de réalités humaines où la "conscience diasporique " garde une place cardinale. Pour ma part, j'ai choisi d'utiliser l'expression "diaspora vietnamienne» pour désigner cette réalité complexe et hétérogène que constituent des populations vietnamiennes dispersées aujourd'hui dans le monde. Ce choix s'explique par le fait qu'elle correspond au mieux à ce qui caractériserait aujourd'hui cette population: des motifs de déplacement, une conscience collective identitaire, une mémoire collective, le maintien des relations entre des groupes éloignés, un désir/ une orientation "vers -le pays- natal- malgré- l'éloignement ", etc. alors que ces caractéristiques sont absentes ou floues dans d'autres expressions. Par ailleurs, « diaspora vietnamienne » offre l'avantage de faire prendre conscience de cette nouvelle réalité qu'est l'émergence d'un transnationalisme vietnamien dans le contexte de la globalisation ${ }^{19}$. Dans cette perspective, ma thèse s'intéresse non seulement à la « diaspora vietnamienne» au sens restrictif du terme, mais aussi à la catégorie des étudiants vietnamiens à l'étranger dont je vais expliquer l'intérêt scientifique dans la partie suivante.

\section{Etudiants vietnamiens à l'étranger : intérêt d'un regard anthropologique et sociologique sur la figure du « migrant connecté »}

6 En effet, cette population étudiante spécifique en nombre croissant offre un terrain d'analyse intéressant pour les raisons suivantes :

- si, par le passé, le phénomène des étudiants vietnamiens partant étudier à l'étranger est déjà un fait historique connu ${ }^{20}$, le récent mouvement de déplacement de cette population étudiante vietnamienne à l'étranger fin $\mathrm{XX}^{\mathrm{e}}$ et début $\mathrm{XXI}^{\mathrm{e}}$ siècles constitue un phénomène d'ordre socioculturel spécifique lié au contexte de la globalisation ; 
- à la différence des «Viet kieu », les étudiants vietnamiens contemporains à l'étranger sont encore très peu étudiés en sciences sociales et humaines ;

- inscrite d'emblée dans la problématique centrale du lien entre le Vietnam et la diaspora, la figure des étudiants vietnamiens à l'étranger permettra d'appréhender la reconfiguration culturelle et identitaire du Vietnam contemporain sous l'angle d'une pratique imaginative à l'œuvre dans les TIC.

7 Ce phénomène de la mobilité internationale des étudiants vietnamiens connait depuis une dizaine d'années une croissance considérable. À la différence de l'immigré « qui arrive pour rester » dans un pays autre que le Vietnam, ces étudiants rentrent dans la catégorie de migrants «une personne en transit, qui vient seulement pour travailler, traverse nos territoires, nos villes et qui retourne à la maison ou repart ailleurs » (Diminescu, 2007). Etant « acteurs d'une culture de lien, qu'ils ont eux-mêmes fondée et qu'ils entretiennent dans la mobilité ", utilisant massivement les TIC, ils rendent visible et dynamique cette culture du lien, car ils sont capables de «maintenir à distance et à activer quotidiennement des relations qui s'apparentent à des rapports de proximité » (Diminescu, 2007). Témoignant d'une nouveauté dans l'histoire de la migration contemporaine: «Hier: immigrer et couper les racines; aujourd'hui : circuler et garder le contact ", ils marquent la naissance de «l'âge du migrant connecté », incarnent cette figure de "l'entre-deux» et attestent la validité de la vulgate " ni ici, ni là-bas, mais ici et là-bas en même temps » (Diminescu, 2007). En résumé, d'un point de vue actanciel, l'analyse des acteurs contemporains de la mobilité, celle des Vietnamiens diasporiques nous permettra de saisir une caractéristique fondamentale du monde contemporain : la circulation, bien plus que les structures et les organisations stables (Appadurai 1996, Urry 2005, Diminescu 2007). À ces flux globaux « ethnoscapes », s'ajouteront des flux " mediascapes ", selon la vision appaduraïenne du paysage de la globalisation, dans lequel est déjà entré le Vietnam contemporain. Mais ces flux globaux n'étant pas indépendants «des structures politico-économiques nationales et transnationales qui facilitent, canalisent et contrôlent les flux de populations, de marchandises et d'idées $»^{21}$, il est indispensable de les contextualiser, de les situer dans des structures de pouvoir globales ainsi que dans les stratégies de l'État. D'où la nécessité, dans le cadre de ma thèse, d'analyser le rôle du gouvernement vietnamien en tant qu'acteur public qui agit à travers des « dispositifs de pouvoir ", au sens foucaldien du terme, pour tenter de réguler ces flux globaux. La question de dispositif, celle de la dimension technique, matérielle du phénomène de l'imaginaire social et de la reconfiguration identitaire est cruciale et indissociable de la question du sens. C'est à ce double aspect qu'est consacrée la partie suivante.

\section{Question de dispositifs et de sens : TIC comme lieux et pratiques de l'imagination collective et sociale}

8 À chaque catégorie d'acteurs correspondent le choix d'un type de dispositifs et une construction de sens (symbolique). Comme ma recherche repose sur l'hypothèse du rôle majeur que jouent les TIC dans la construction de l'imaginaire social du Vietnam contemporain, mon corpus se concentrera sur quelques exemples de phénomènes Internet relatifs à la thématique des liens entre Vietnamiens nationaux et Vietnamiens diasporiques. Dans le cadre de cette communication, mon analyse se limite à deux exemples: la chanson «Bonjour Vietnam»et les manifestations anti-chinoises des Vietnamiens du pays et de la diaspora quant au contentieux des archipels des Spratly et 
des Paracels. Dans chaque cas, je présenterai une approche méthodologique et j'expliquerai les enjeux liés à la problématique de ma recherche.

\section{La chanson « Bonjour Vietnam »}

Voici un mot sur l'histoire: Pham Quynh Anh, une jeune belge d'origine vietnamienne a eu l'occasion au début de 2005 de chanter en duo avec Marc Lavoine, un compositeur français. Ce dernier, touché par le charme innocent et les sentiments intimes d'une vietnamienne née à l'étranger et qui n'avait jamais vu le pays natal de ses parents, a coécrit pour elle la chanson Bonjour Vietnam. "Par erreur, une maquette du titre s'est en effet retrouvée sur Internet. Quelques jours plus tard, la communauté vietnamienne du monde entier entendait "Bonjour Vietnam» et était très touchée par le message "22. Depuis le Têt 2006, Nouvel An traditionnel vietnamien, de forum en forum sur Internet, traduite en vietnamien, puis en anglais, elle a suscité de vives réactions favorables chez des publics très divers, qu'ils soient Vietnamiens diasporiques (Viet kieu et Vietnamiens expatriés) ou amoureux du Vietnam. La chanson a fait le tour du monde " virtuel » pour " arriver " au Vietnam où elle a été chaleureusement saluée par les mass médias officiels du gouvernement. En circulant sur la Toile, la chanson a fait l'objet de transformations multiples depuis plus de deux ans, au gré de l'imagination des internautes et de leur compétence en matière d'informatique : de fichier audio au format diaporama, jusqu'au vidéoclip sur Youtube et Dailymotion. Enfin, en avril 2008, le CD single «Bonjour Vietnam» est sorti officiellement, mais en version anglaise «Hello Vietnam», chez iTunes. Ici, j'adopterai une analyse de contenu (du texte de la chanson) et une analyse sémiotique de ses divers formats matériels. Cette double approche s'attachera à mettre au jour la dimension triviale de la culture, particulièrement visible dans ce phénomène : il s'agit de saisir «cette force de la composante triviale de la culture, ce rôle que jouent les circuits, très petits ou mondiaux, par lesquels une cuvre devient un objet partagé et détourné, par lesquels les idées se font texte circulant » (Jeanneret, 2008, p. 252). L'analyse consistera à considérer ce qui a favorisé la diffusion de la chanson et a permis de la transformer au gré des circonstances : texte, images, supports techniques, acteurs de la diffusion, circuits de la circulation, institutions de légitimation, idéologie et imaginaire que construit ou véhicule cette chanson. Elle souligne ainsi à la fois la dimension dynamique et créative (poétique) de la culture qui se forme, se transforme, s'invente et se mêle en circulant et la dimension de l'ordinaire manifesté dans les formes, les supports matériels, les acteurs impliqués. L'enjeu de l'analyse serait de réfléchir sur le phénomène "Bonjour Vietnam » lié à la problématique centrale de la thèse : comment ce phénomène construit, configure et rend$\mathrm{t}$-il visible la dimension transnationale du Vietnam contemporain, en connectant des Vietnamiens nationaux aux Vietnamiens diasporiques? Comment produit-il des discours symboliques à caractère à la fois identitaire et inventif ? La dernière question renvoie à la question de sens fortement manifeste dans ce phénomène identitaire ${ }^{23}$.

\section{Manifestations anti-chinoises relatives au contentieux des archipels des Spratly et des Paracels}

Voici un mot sur l'histoire: le 4 décembre 2007, les médias officiels vietnamiens donnent des nouvelles concernant l'opposition du Vietnam contre la création du gouvernement chinois de la municipalité de Sansha incluant les deux archipels vietnamiens, des Spratleys et des Paracels. Les bloggeurs vietnamiens importants sur Yahoo 360 ont 
commencé à faire appel à des manifestations publiques à Hanoi et Ho Chi Minh Ville. Le 9 décembre, la première manifestation s'est déroulée devant l'ambassade de Chine à Hanoi et son Consulat à Ho Chi Minh Ville, rassemblant surtouts des étudiants. Entre les 10 et le 15, des bloggeurs sur Yahoo 360 ont discuté en détail ces événements et appelé à plus de manifestations pour le 16. Les forces de l'ordre ont essayé d'intervenir. Le 16 décembre, la deuxième manifestation a eu lieu à Hanoi et à Ho Chi Minh Ville, à une dimension plus restreinte, à cause de l'intervention des forces de l'ordre. Le 20 décembre, les autorités vietnamiennes ont demandé à la population de ne plus manifester contre la Chine. Entre temps, le 12 décembre, un article de Vietnamnet, un website d'information officiel, a fait l'éloge de la manifestation du 9 décembre, considérée comme patriotique. Son rédacteur en chef, alors en déplacement professionnel, a été promptement réprimandé. Le 14 décembre, certains chercheurs et universitaires vietnamiens ont joint leurs forces pour créer une fondation (South East Asia Sea Research Foundation) afin d'éveiller la conscience du monde entier et de soutenir des recherches sur la Mer du Sud-est asiatique. À l'extérieur du Vietnam, l'événement a aussi fortement affecté des communautés vietnamiennes diasporiques : les étudiants vietnamiens se sont manifestés contre la Chine un peu partout en Europe pendant décembre 2007 et janvier 2008 (Paris, Londres, Berlin, Prague, Bruxelles). Ici, il s'agit d'analyser, dans le paysage des mediascapes vietnamiens, l'articulation entre le nouveau phénomène des blogs et l'expression possible d'une pluralité de constructions du réel à travers ce grand événement sociopolitique. L'approche sémiotechnique servira à appréhender comment les blogs en tant que nouveaux dispositifs (outil de publication et nouveaux medias), appropriés par divers acteurs vietnamiens, notamment les étudiants, ont créé une autre optique de la réalité que celle des médias mainstream strictement contrôlés par le gouvernement vietnamien. Dépassant de loin la fonction d'un journal intime sur Internet, la blogosphère a opéré un grand impact médiatique de mobilisation auprès des Vietnamiens aussi bien au Vietnam qu'en diaspora. Non seulement liée aux configurations techniques du dispositif, la force mobilisatrice de cette action sociale tient également à la nature identitaire de l'événement. L'enjeu consiste à saisir comment cette vague technologique, qui affecte à la fois adolescents, étudiants, intellectuels, artistes et professionnels de tout âge, remet en question l'économie des médias traditionnels au Vietnam, reconfigure les liens entre Vietnamiens nationaux et diasporiques, et construit ainsi un nouvel imaginaire social du Vietnam.

\section{Conclusion}

11 À partir d'une posture épistémologique communicationnelle, mon approche méthodologique a établi des liens avec d'autres sciences humaines et sociales (sociologie, anthropologie, sciences politiques, sémiotique, histoire...) de manière à tenter d'apporter un nouvel éclairage sur la problématique de l'imaginaire social du Vietnam. Cette construction du dispositif méthodologique a conféré à mon objet de recherche quelques spécificités: concentration sur la question du sens et de sa circulation (discours) ; prise en compte des acteurs en jeu en réhabilitant la place des Vietnamiens nationaux dans ce processus transnational ; importance accordée aux aspects technologiques du phénomène identitaire / imaginaire. Ici, l'approche communicationnelle de l'imaginaire social permettrait d'éviter une double conception réductrice de la communication: comme un modèle normatif de transfert d'informations ou comme un processus d'échange de 
significations intentionnellement définies (Jeanneret, 2008). Il existe un enjeu de penser l'acte de communication comme composante nécessaire à l'appropriation des objets culturels, à leur existence sociale : elle "ne se bornera pas à transmettre du social, du symbolique déjà existants, mais elle en engendre». Au fond c'est "une interrogation sur la nature du pouvoir que les hommes en société exercent sur leur environnement culturel » (Jeanneret, 2008, p.17). Toute constitution du dispositif méthodologique fait partie intégrante d'un processus plus général de construction d'un objet ou d'une problématique de recherche. Dans cette perspective, tenter d'étudier la question de l'imaginaire social du Vietnam contemporain en adoptant une posture interdisciplinaire des SIC consiste à se donner un défi scientifique, celui de produire un nouvel éclairage sur un phénomène complexe, c'est-à-dire celui de produire de la connaissance.

\section{BIBLIOGRAPHIE}

Appadurai, A., (2001), Après le colonialisme : Les conséquences culturelles de la globalisation, Payot, 334p.

Diminescu, D., (2007), Le migrant connecté - pour un manifeste épistémologique, in Migrations/ Société, vol.17, n 102, 2007, pp. 275-292.

Dorais, L.-J., (2001), Defining the Overseas Vietnamese, in Diaspora, 30 (1), 2001, pp. 3-27.

Jeanneret, Y., (1998), L'affaire Sokal ou la querelle des impostures, Presses Universitaires de France, $274 \mathrm{p}$.

Jeanneret, Y., (2008), Penser la trivialité. Volume 1 : la vie triviale des êtres culturels, Hermès Science, Lavoisier, 267p.

Le, H. K., (1985), Les Vietnamiens en France: insertion et identité, Paris, L'Harmattan, 297p.

Le, S.L., The Global Vietnamese Diaspora, http://blogs.bauer.uh.edu/vietDiaspora, date de la dernière visite : 13 juin 2008 .

Nguyen, B. T., Cộng Đồng Người Việt Xa Quê Hương: Vấn Đề Nhân Số và Tên Gọi [Communauté des Vietnamiens résidant à l'étranger : question de nombre et de nom], http://www.dunglac.net/ bai/NgBTung-01-cdviet.htm, date de la dernière visite : 13 juin 2008.

Ollivier, B., (2007), Les Sciences de la communication, Armand Colin, 284p.

Ricœur, P., (1991), The Creativity of Language: Interview with Richard Kearney, in M.J. Valdes (dir.), A Ricœur Reader: Reflection and Imagination, Toronto, University of Toronto Press, pp. 463-481.

Tölölyan, K., (1996), Rethinking diaspora(s): Stateless power in the transnational moment, in Diaspora, vol. 5, $\mathrm{n}^{\circ} 1, \mathrm{pp} .3-36$. 


\section{NOTES}

1. Titre provisoire de la thèse: Liens entre le Vietnam et la diaspora: cyber-Vietnamité ou construction d'un imaginaire social du Vietnam contemporain à travers des technologies de de l'information et de la communication.

2. H. K. LE, 1985.

3. L.-J. DORAIS, 1998, 2001, 2004 ; L.-J. DORAIS et E. RICHARD, 2007.

4. S. L. LE, 2008.

5. Ibid.

6. R. M. Abrami, G. Pringle, « Viet kieu Word Origin », archives des listes de discussions sur le site de Vietnam Studies Group, 2001, http://www.lib.washington.edu/southeastasia/vsg/elist_2001/ Vietkieu.html, date de la dernière visite : 13 juin 2008.

7. Idem, Nhung Tuyet Tran.

8. Ibid, Nhung Tuyet Tran.

9. Ibid, Cong Huyen Ton Nu Nha Trang.

10. "Hội Việt kiều yêu nước".

11. Resolution No 36 - NQ/TW, March 26, 2004, http://www.quehuong.org.vn/en/nr050307123954/ nr050314142833/ns050315100202, date de la dernière visite : 13 juin 2008.

12. Decision No 135/2007/QD-TTg dated August 17, 2007 by the Prime Minister on The Promulgation of the Regulation on Visa Exemption for Vietnamese Residing Overseas, http:// www.quehuong.org.vn/en/nr050307123954/nr070829151334/, date de la dernière visite : 13 juin 2008.

13. Résolution No 36- NQ/TW du 26 mars 2004 du Bureau politique relative au travail vis à vis des Vietnamiens résidant à l'étranger et Arrêté No 135/2007/QD-TTG du Premier Ministre, en date du 17 août 2007 portant publication du Règlement relatif à l'exemption de visa en faveur des Vietnamiens résidant à l'étranger. http://www.quehuong.org.vn/fr, date de la dernière visite : 13 juin 2008.

14. B. T. Nguyen, 2003. Ma traduction.

15. Voir L. Anteby-Yemini, Les Diasporas : 2000 ans d'histoire, 2005.

16. Cette hypothèse est avancée par le chercheur N. B. Tung, 2003. Il cite l'exemple de « Mouvement des Laïcs Vietnamiens de la Diaspora » (The Vietnamese Laity Movement in Diaspora).

17. B. T. Nguyen, 2003.

18. Tölölyan propose les critères suivants pour caractériser une diaspora: une dispersion forcée par contraintes politiques, économiques ou autres ; le partage d'une identité bien définie avant la dispersion; le maintien ou la construction d'une mémoire collective; capacité de garder un contrôle de leur frontière ethnique; le désir et le fait de maintenir des relations entre communautés diasporiques et celles avec le pays d'origine; la présence de valeurs et d'organisations transnationales collectives. Voir Tölölyan, 1996.

19. Voir Cohen, Global Diasporas, 1997 et Van Hear, New Diasporas, 1998.

20. S. Mc Connell, Leftward Journey: The Education of Vietnamese Students in France, 1919-1939; V. Daniel, L'Identité narrative des jeunes Vietnamiens en France, thèse de doctorat, 1996, Université de Nice Sophia-Antipolis.

21. A. Ong, Flexible Citizenship. The Cultural Logics of Transnationality, Duke University Press, Durham, 1999, p.11.

22. L'explication est donnée par Pham Quynh Anh elle-même sur son site officiel: http:// quynhanh-music.artistes.universalmusic.fr/main_site.php, date de la dernière visite : 13 juin 2008. 
23. Sur le rôle particulier que joue la musique dans la création d'une culture transnationale vietnamienne, voir la thèse de $\mathrm{C}$. Kieu Linh Valverde: Making Transnational Vietnam: Vietnamese Americans Community-Vietnam Linkages through Money, Music and Modems, University of California, Berkely, 2002.

\section{RÉSUMÉS}

Cette communication, dont l'enjeu est d'ordre épistémologique, s'attache à s'interroger en quoi l'approche communicationnelle permettrait d'apporter un éclairage neuf sur la vieille question de l'identité culturelle vietnamienne vue sous l'angle d'un imaginaire social. Après avoir exposé la problématique de recherche, celle de la cyber-vietnamité conçue comme un imaginaire social, au sens ricœurien, supposé à l'œuvre aujourd'hui dans les liens entre les Vietnamiens nationaux et la diaspora, par le biais des médias informatisés, cette étude explicite la spécificité du regard communicationnel : l'articulation de trois dimensions de nature différente, à savoir celle de la technique, celle du social et celle du sens, pour appréhender cet objet hétérogène et composite. D'où le choix d'une triple approche: une approche logistique (TIC comme dispositifs de construction), une approche actancielle (des Vietnamiens nationaux et diasporiques), et une approche herméneutique (sens donnés à cet imaginaire social).

This paper, which aims at articulating an epistemological reflection, attempts to question the way in which a communicational approach will throw a new light on the old topic of Vietnamese cultural identity, regarded as a social imaginary. After stating the research issue - a "cybervietnamness" conceived as a social imaginary, in Ricœur's understanding of the phrase, supposed to be at work in today's links between Vietnam and its diaspora through technologies of information and communication (TIC) - I will clarify the specificity of communicational posture: simultaneous articulation of three dimensions, namely logistic (TIC as material, technical means), social (national and diasporic Vietnamese people) and symbolic (meanings attributed to that social imaginary), of that heterogeneous and composite object.

\section{INDEX}

Mots-clés : approche communicationnelle, imaginaire social, Vietnam, discours, diaspora, TIC, pratique

Keywords : communicational approach, social imaginary, Vietnam, speech, dispora, Information and Communication Technology, practice

\section{AUTEUR}

\section{ANH-NGOC HOANG}

Anh Ngoc Hoang a travaillé comme enseignant de français et interprète-traducteur au Vietnam (1999-2004). En 2004, il intègre le CELSA, Université Paris IV où il a fait son mémoire de Master intitulé « Processus de construction des imaginaires d'Internet au Vietnam: Internet comme 
utopie et idéologie de la modernité? ». Sa thèse doctorale, inscrite dans le cadre du GRIPIC (Groupe de Recherches Interdisciplinaires sur les Processus d'Information et de Communication), est intitulée « Liens entre le Vietnam et la diaspora : cyber-vietnamité. Construction médiatique d'un imaginaire social du Vietnam contemporain à travers des médias informatisés ». 